\title{
3 Research Square

\section{Which combination is best for HbA1c, fasting plasma glucose, and 2-h post-challenge glucose for diagnosis of diabetes mellitus}

Junichi Okada

Gunma Daigaku Daigakuin Igakukei Kenkyuka Igakubu

Kohji Kashima

Kiryu Kosei Sogo Byoin

Eijiro Yamada

Gunma Daigaku Igakubu Fuzoku Byoin

Yasuyo Nakajima

Gunma Daigaku Igakubu Fuzoku Byoin

Kazuya Okada

Omagari Kousei Medical Center

Shuichi Okada ( $D$ okadash@gunma-u.ac.jp )

Gunma Daigaku Igakubu Fuzoku Byoin https://orcid.org/0000-0003-1403-6840

Masanobu Yamada

Gunma Daigaku Daigakuin Igakukei Kenkyuka Igakubu

Research article

Keywords: Diabetes mellitus, Fasting plasma glucose, $75 \mathrm{~g}$ oral glucose tolerance test

Posted Date: July 23rd, 2020

DOI: https://doi.org/10.21203/rs.3.rs-46159/v1

License: (c) (i) This work is licensed under a Creative Commons Attribution 4.0 International License.

Read Full License 


\section{Abstract \\ Background}

We evaluated reliability among glycated hemoglobin (HbA1c), fasting plasma glucose (FPG), and a $75 \mathrm{~g}$ oral glucose tolerance test ( $75 \mathrm{~g}$ OGTT) where 2-h post-challenge glucose level ( $2 \mathrm{hPG}$ ) in the diagnosis of diabetes and pre-diabetes to find out which parameter or combination of parameters is useful.

\section{Methods}

All the participants received $75 \mathrm{~g}$ OGTT and $\mathrm{HbA} 1 \mathrm{c}$ measurements. For FPG,$<110 \mathrm{mg} / \mathrm{dL}$ was defined as "normal", $110 \leq \mathrm{FPG}<126 \mathrm{mg} / \mathrm{dL}$ was defined as "pre-diabetes", and $126 \mathrm{mg} / \mathrm{dL} \leq \mathrm{FPG}$ was defined as "diabetes". For $2 \mathrm{hPG},<140 \mathrm{mg} / \mathrm{dL}$ was defined as "normal", $140 \mathrm{mg} / \mathrm{dL} \leq 2 \mathrm{hPG}<200 \mathrm{mg} / \mathrm{dL}$ was defined as "pre-diabetes", and $200 \mathrm{mg} / \mathrm{dL} \leq$ was defined as "diabetes". For HbA1c, $\leq 6.2 \%$ was defined as "normal", $6.2<\mathrm{HbA} 1 \mathrm{c}<6.5 \%$ was defined as "pre-diabetes", and $6.5 \% \leq$ was defined as "diabetes". The prevalence of diabetes and pre-diabetes were estimated based on 7 different combinations as the following: "the combination of either HbA1c or FPG or $2 \mathrm{hPG}$ ", "the combination of either HbA1c or $2 \mathrm{hPG",}$ "the combination of either HbA1c or FPG", "HbA1c alone", "the combination of either FPG or 2hPG", "2hPG alone", and "FPG alone". Each diagnosed group were then divided into different age groups (40 s, $50 \mathrm{~s}, 60 \mathrm{~s}, 70 \mathrm{~s}$, and $80 \mathrm{~s}$ ) to consider the effect of age on results.

\section{Results}

For diagnosing "diabetes", "the combination of either HbA1c or $2 \mathrm{hPG"} \mathrm{was} \mathrm{almost} \mathrm{as} \mathrm{reliable} \mathrm{as} \mathrm{"the}$ combination of either $\mathrm{HbA} 1 \mathrm{c}$ or FPG or $2 \mathrm{hPG}$ " throughout all ages except for the $50 \mathrm{~s}$. For diagnosing "pre-diabetes", "the combination of either $\mathrm{HbA1c}$ or $2 \mathrm{hPG}$ " was almost as reliable as "the combination of either $\mathrm{HbA} 1 \mathrm{c}$ or $\mathrm{FPG}$ or $2 \mathrm{hPG}$ " throughout all ages.

\section{Discussion}

Combining our results, measurement of $\mathrm{HbA} 1 \mathrm{c}$ and $2 \mathrm{hPG}$ seems to be recommended for the diagnosis of diabetes and pre-diabetes. This means for an accurate diagnosis of diabetes and pre-diabetes, not only the measurement of $\mathrm{HbA1c}$ but also a $75 \mathrm{~g}$ OGTT is recommended. Unfortunately, screening by FPG alone is not recommended from this clinical study.

\section{Conclusions}

For accurate diagnosis of diabetes and pre-diabetes, the combination of $\mathrm{HbA} 1 \mathrm{c}$ measurement and $75 \mathrm{~g}$ OGTT seemed to be a secure concept. 


\section{Introduction}

Fasting plasma glucose (FPG) has been used as a criterion for diagnosing diabetes, alone or during a $75 \mathrm{~g}$ oral glucose tolerance test (75 g OGTT) where 2-h post-challenge glucose level ( $2 \mathrm{hPG}$ ) is also a criterion. This method is sensitive, but has poor reproducibility because of day to day variation. In recent years, glycated hemoglobin (HbA1c), initially discovered by Rahbar et al in 1969 [1], was recommended as a tool for the diagnosis and monitoring of treatment of diabetes. In 2009, the International Expert Committee recommended using $\mathrm{HbA} 1 \mathrm{c} \geq 6.5 \%$ to diagnose diabetes [2], and this position was also endorsed by the American Diabetes Association [3]. In this study, we sought to evaluate the concordance among $\mathrm{HbA} 1 \mathrm{c}, \mathrm{FPG}$, and $2 \mathrm{hPG}$ in the diagnosis of pre-diabetes (containing impaired fasting glucose; IFG, impaired glucose tolerance; IGT, and IFG + IGT; combined glucose intolerance) and diabetes to find out which parameter or combination of parameters is useful.

\section{Method And Subjects}

The study protocol was reviewed and approved by our hospital review board according to the guidelines of the Declaration of Helsinki. A written informed consent was obtained from each participant. This clinical study consisted of 3,681 subjects who received comprehensive medical checkups at Kiryu Kosei General Hospital. All the subjects did not receive any medication to treat hypertension or hyperlipidemia etc. The subjects who had already been diagnosed with diabetes mellitus or who were taking diabetes medications were excluded from this study.

The study subjects were made to fast overnight and their blood samples were drawn at 8 o'clock in the morning and 2 hours after oral administration of $75 \mathrm{~g}$ glucose as $75 \mathrm{~g}$ OGTT. Venous blood samples were collected into tubes containing EDTA and fluoride. Plasma was separated from the cells within $<1$ hour after withdrawal, and glucose concentrations in plasma were determined by a hexokinase method using a Synchro CX4/CX5 glucose analyzer (Beckman Instruments, Fullerton, CA). Both the intra- and interassay coefficients of variation were $\leq 2 \%$ at values $<7 \mathrm{mmol} / \mathrm{L}$.

$\mathrm{HbA1c}$ was assessed in blood samples collected on EDTA using the Bayer DCA 2000 device (Bayer Diagnostics Puteaux, France). This uses an immunological method to measure HbA1c. Normal values range from 4 to $6 \%$. We also used the HPLC method (Biorad DIAMAT, Ivry-sur-Seine, France) to determine $\mathrm{HbA} 1 \mathrm{c}$ concentrations. Normal values range from 4.5 to $6.5 \%$.

All the participants received $75 \mathrm{~g}$ OGTT and $\mathrm{HbA} 1 \mathrm{c}$ measurements. For FPG,$<110 \mathrm{mg} / \mathrm{dL}$ was defined as "normal", $110 \leq \mathrm{FPG}<126 \mathrm{mg} / \mathrm{dL}$ was defined as "pre-diabetes", and $126 \mathrm{mg} / \mathrm{dL} \leq \mathrm{FPG}$ was defined as "diabetes". For $2 \mathrm{hPG},<140 \mathrm{mg} / \mathrm{dL}$ was defined as "normal", $140 \mathrm{mg} / \mathrm{dL} \leq 2 \mathrm{hPG}<200 \mathrm{mg} / \mathrm{dL}$ was defined as "pre-diabetes", and $200 \mathrm{mg} / \mathrm{dL} \leq$ was defined as "diabetes". For HbA1c, $\leq 6.2 \%$ was defined as "normal", $6.2<\mathrm{HbA} 1 \mathrm{c}<6.5 \%$ was defined as "pre-diabetes", and $6.5 \% \leq$ was defined as "diabetes".

The prevalence of diabetes and pre-diabetes were estimated based on 7 different combinations as the following: "the combination of either HbA1c or FPG or $2 \mathrm{hPG}$ ", "the combination of either HbA1c or $2 \mathrm{hPG}$ ", 
"the combination of either HbA1c or FPG", "HbA1c alone", "the combination of either FPG or 2hPG", "2hPG alone", and "FPG alone". Each diagnosed group were then divided into different age groups (40 s, $50 \mathrm{~s}, 60 \mathrm{~s}, 70 \mathrm{~s}$, and $80 \mathrm{~s}$ ) to consider the effect of age on results.

\section{Results}

Subjects background was as follows: Male/Female was at 2.3/1. Age was at $55.2 \pm 10.7$ (years old, mean \pm standard deviation), Body length was at $165 \pm 8.9 \mathrm{~cm}$. Body weight was at $62.3 \pm 11.8 \mathrm{~kg}$. Body mass index was at $22.8 \pm 3.2\left(\mathrm{~kg} / \mathrm{m}^{2}\right)$. Systolic pressure was at $120.1 \pm 15 \mathrm{mmHg}$. Diastolic pressure was at $74.4 \pm 9.6 \mathrm{mmHg}$. Waist circumference was at $83.4 \pm 8.8 \mathrm{~cm}$. Serum creatinine was at $0.78 \pm$ $0.26 \mathrm{mg} / \mathrm{dL}$. HDL cholesterol was at $64.3 \pm 16.8 \mathrm{mg} / \mathrm{dL}$. LDL cholesterol was at $128.9 \pm 29 \mathrm{mg} / \mathrm{dL}$.

Triglyceride was at $208.8 \pm 31.4 \mathrm{mg} / \mathrm{dL}$. Uric acid was at $5.6 \pm 1.3 \mathrm{mg} / \mathrm{dL}$. Fasting plasma glucose was at $92.9 \pm 7.6 \mathrm{mg} / \mathrm{dL}$. $2 \mathrm{hPG}$ was at $107.3 \pm 18.8 \mathrm{mg} / \mathrm{dL}$. HbA1c was at $5.55 \pm 0.25 \%$. Of the 3,681 subjects, $624,1410,1177,374$, and 96 were in their 40 s, 50 s, 60 s, 70 s, and 80 s, respectively.

The diagnosis of "diabetes", "pre-diabetes", and "normal" were made using different combinations of $\mathrm{HbA1c}, \mathrm{FPG}$, and $2 \mathrm{hPG}$. We presented the number of subjects assigned to each diagnosis when using "the combination of either HbA1c or FPG or $2 \mathrm{hPG}$ " as $100 \%$. We then estimated the efficiency of diagnosis with different combinations by comparing each combination to "the combination of either $\mathrm{HbA} 1 \mathrm{c}$ or $\mathrm{FPG}$ or $2 \mathrm{hPG}$ ".

When diagnosing "diabetes", "the combination of either HbA1c or $2 \mathrm{hPG}$ " was almost as reliable as "the combination of either HbA1c or FPG or 2 hPG" throughout all ages except for the $50 \mathrm{~s}$ (Fig. 1-i). However, none of the combinations of "the combination of either HbA1c or FPG", "HbA1c alone", "the combination of either FPG of $2 \mathrm{hPG}$ ", "2hPG alone", nor "FPG alone" were reliable to diagnose diabetes since the values were below 100 (Fig. 1a).

When diagnosing "pre-diabetes", "the combination of either HbA1c or 2hPG" was almost as reliable as "the combination of either HbA1c or FPG or 2hPG" throughout all ages (Fig. 1b). However, none of the combinations of "the combination of either HbA1c or FPG", "HbA1c alone", "the combination of either FPG of $2 \mathrm{hPG}$ ", "2hPG alone", nor "FPG alone" were reliable to diagnose pre-diabetes since the values were below 100 (Fig. 1b).

However, in the case of diagnosing "normal", none of the combinations of "the combination of either $\mathrm{HbA} 1 \mathrm{c}$ or $2 \mathrm{hPG}$ ", "the combination of either HbA1c or FPG", "HbA1c alone", "the combination of either FPG of 2hPG", "2hPG alone", nor "FPG alone" were reliable (Fig. 1c). These factors seemed to overdiagnose because their values were beyond 100 suggesting that either pre-diabetes or diabetes subjects were counted as normal throughout all ages.

\section{Discussion}


FPG has been used as a criterion for diagnosing diabetes, alone or during a $75 \mathrm{~g}$ oral glucose tolerance test (75 g OGTT) where 2-h post-challenge glucose level ( $2 \mathrm{hPG}$ ) is also a criterion. In recent years, $\mathrm{HbA} 1 \mathrm{c}$ was recommended as a tool for the diagnosis and monitoring of treatment of diabetes. However, it is known that different diagnostic measures have different sensitivities. It is also known that HbA1c increases with age and the sensitivity of $\mathrm{HbA} 1 \mathrm{c}$ for diagnosis of diabetes compared to OGTT is quite limited. Therefore, we decided to determine which factor or combination of factors among HbA1c, FPG, and $2 \mathrm{hPG}$ is the most reliable for diagnosing pre-diabetes and diabetes.

In this paper, 7 combinations among $\mathrm{HbA} 1 \mathrm{c}$, FPG, and $2 \mathrm{hPG}$ were compared for the diagnosis of diabetes and pre-diabetes. For the diagnosis of diabetes, either $6.5 \% \leq \mathrm{HbA} 1 \mathrm{c}$ or $126 \mathrm{mg} / \mathrm{dL} \leq \mathrm{FPG}$ or $200 \mathrm{mg} / \mathrm{dL}$ $\leq 2 \mathrm{hPG}$ was used. For diagnosis of pre-diabetes, either $6.2<\mathrm{HbA} 1 \mathrm{c}<6.5 \%$ or $110<\mathrm{FPG}<126$ or $140<$ $2 \mathrm{hPG}<200$ was used. Therefore, normal subjects were obtained by subtraction of diabetes and prediabetes from the enrolled subjects. The diagnosis of "diabetes", "pre-diabetes", and "normal" were made using different combinations of HbA1c, FPG, and 2hPG, as shown in the "Methods and Subjects".

Since "the combination of either HbA1c or FPG or 2hPG" (one of three factors were fit to diabetes criterion) is the most reliable criterion because it includes $\mathrm{HbA} 1 \mathrm{c}$ to minimize the day to day variation, we considered and represented the incidence of diabetes by using "the combination of either HbA1c or FPG or $2 \mathrm{hPG}$ " as $100 \%$. Thereafter, incidence of diabetes from the rest of combinations was represented as a relative ratio to "the combination of either $\mathrm{HbA} 1 \mathrm{c}$ or $\mathrm{FPG}$ or $2 \mathrm{hPG}$ ". In this case, when the value is below 100 , it means the combination includes false negative. When the value is above 100 , it means the combination includes false positive.

Based on this design, we found that "the combination of either HbA1c or $2 \mathrm{hPG}$ " (one of two was fit to diabetes criterion) had comparable reliability to "the combination of either HbA1c or FPG or 2hPG" throughout all ages except for the $50 \mathrm{~s}$. The rest of the combinations of factors were not reliable compared to "the combination of either HbA1c or FPG or 2hPG" throughout all ages including "the combination of either HbA1c or FPG", or "HbA1c alone", or "FPG alone" which has been used currently for diagnosis of diabetes as described above [2,3]. Combining these results, measurement of $\mathrm{HbA} 1 \mathrm{c}$ and $2 \mathrm{hPG}$ seems to be recommended for the diagnosis of diabetes. This means for an accurate diagnosis of diabetes, not only the measurement of $\mathrm{HbA} 1 \mathrm{c}$ but also a 75gOGTT is recommended. Unfortunately, screening by FPG alone is not recommended from this clinical study, although previously there has been a literature about FPGs usefulness [4].

The screening of pre-diabetes is recently collecting attention from the point of prevention of diabetes mellitus since the STOP NIDDM project [5]. In our study, we found that "the combination of either FPG or $2 \mathrm{hPG}$ " showed equivalent reliability to "the combination of either HbA1c or FPG or $2 \mathrm{hPG}$ ". Our study also showed that neither "the combination of either HbA1c or $2 \mathrm{hPG}$ ", nor "the combination of either HbA1c or FPG", nor "HbA1c alone", nor "2hPG alone", nor "FPG alone" was suitable.

This clinical research has a few limitations that warrant discussion. First, our sample size was small $(\mathrm{n}=$ $3,681)$, so the generalizability of the results is restricted. Therefore, these current results should be verified 
in a larger cohort. Second, the study has a selection bias due to the ethnicity, age, sex, weight, etc., of the participant and would benefit from the inclusion of a range of demographics.

\section{Conclusion}

For accurate diagnosis of diabetes and pre-diabetes, the combination of $\mathrm{HbA} 1 \mathrm{c}$ measurement and $75 \mathrm{gOGTT}$ seemed to be a secure concept.

\section{Abbreviations}

HbA1c; Glycated hemoglobin

FPG; Fasting plasma glucose

$75 \mathrm{~g} \mathrm{OGTT} ; 75 \mathrm{~g}$ oral glucose tolerance test

2 h-PG; $75 \mathrm{~g}$ OGTT where 2-h post-challenge glucose level

\section{Declarations}

\section{Ethics approval and consent to participate}

The study protocol was reviewed and approved by the review board in accordance to the principles of the Declaration of Helsinki. Written informed consent was obtained from the participants prior to publication of this manuscript and any accompanying images. A copy of written consent is available for review by the Editor-in-Chief of this journal.

\section{Consent for publication}

Written informed consent was obtained from the people in each case for publication of this manuscript and any accompanying images. A copy of written consent is available for review by the Editor-in-Chief of this journal.

\section{Availability of data and materials}

The datasets used and/or analyzed during the current study are available from the corresponding author on reasonable request.

\section{Competing interests}

The authors declare that there is no conflict of interest that could be perceived as prejudicing impartiality of the research reported. This research did not receive any specific grant from funding agencies in the public, commercial, or not-for-profit sectors. 


\section{Funding}

The funders had no role in study design, data collection and analysis, decision to publish, or preparation of the manuscript.

\section{Authors' contributions}

JO, KK, EY, YN, KO, SO, and YM collected and interpreted data. SO prepared figures. JO, KO, and MY wrote and edited the manuscript.

\section{Acknowledgements}

This manuscript was edited by MARUZEN Editing services.

\section{References}

1. Rahbar S, Blumenfeld O, Ranney HM. Studies of an unusual hemoglobin in patients with diabetes mellitus. Biochem Biophys Res Commun. 1969;36:838-43.

2. International Expert C. International Expert Committee report on the role of the $\mathrm{A} 1 \mathrm{C}$ assay in the diagnosis of diabetes. Diabetes Care. 2009;32:1327-34.

3. American Diabetes A. Diagnosis and classification of diabetes mellitus. Diabetes Care. 2010;33(Suppl 1):62-9.

4. Expert Committee on the Diagnosis and Classification of Diabetes Mellitus. Follow-up report on the diagnosis of diabetes mellitus. Diabetes Care. 2002;26:3160-7.

5. Chiasson JL, Josse RG, Gomis R, Hanefeld M, Karasik A, Laakso M, STOP-NIDDM Trial Research Group. Acarbose treatment and the risk of cardiovascular disease and hypertension in patients with impaired glucose tolerance: the STOP-NIDDM trial. JAMA. 2003;290:486-94.

6 . Legends.

\section{Figures}




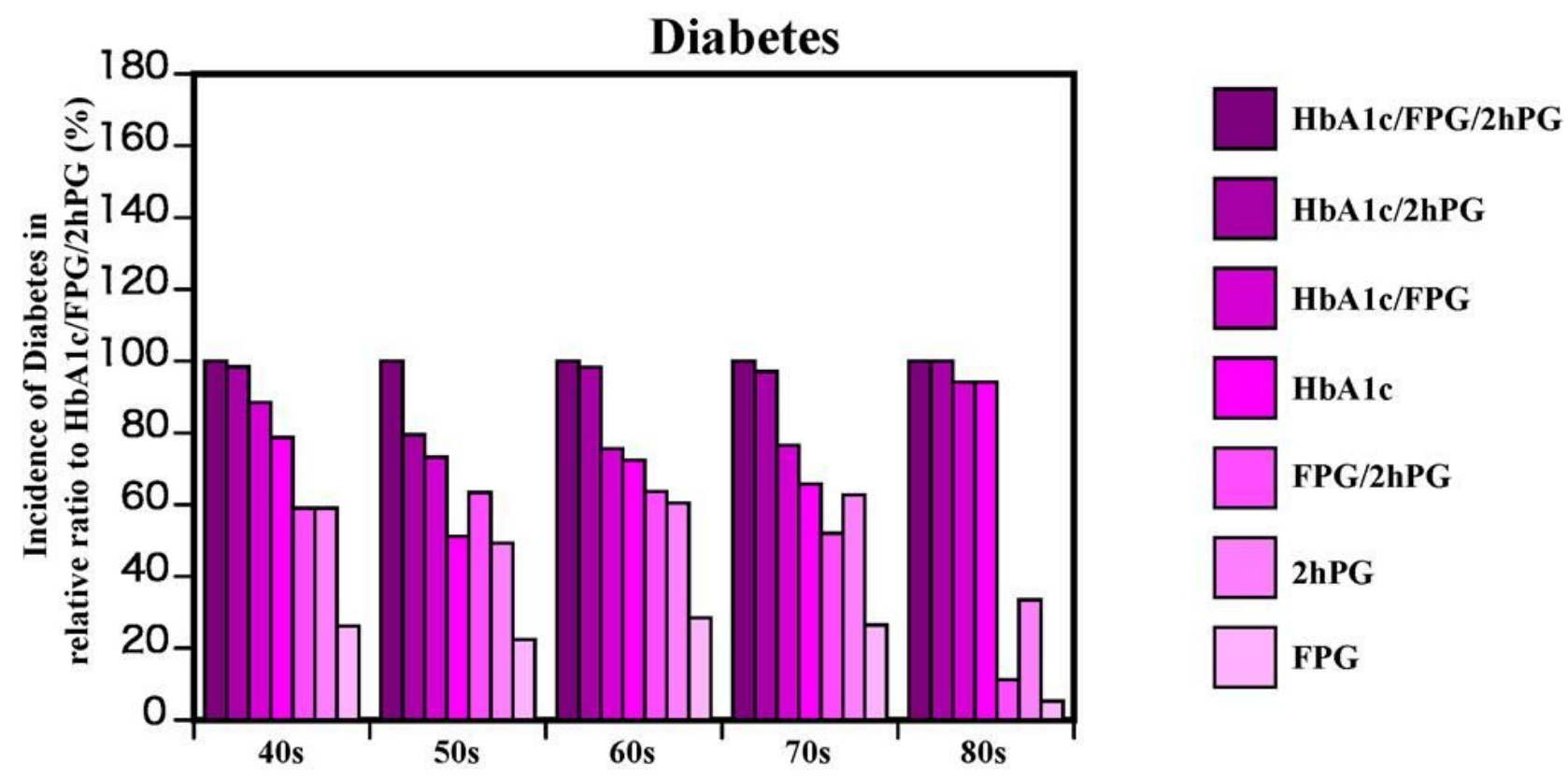

Figure 1

Incidence of Diabetes, Pre-Diabetes, and Normal based on different combinations of HbA1c, FPG, and $2 \mathrm{hPG}$ throughout age groups. a. Incidence of Diabetes. $6.5 \%<\mathrm{HbA} 1 \mathrm{c}$ or $126 \mathrm{mg} / \mathrm{dL}<\mathrm{FPG}$ or $200 \mathrm{mg} / \mathrm{dL}$ $<2 \mathrm{hPG}$ was determined as diabetes. Each combination of these factors was compared to "the combination of either HbA1c or FPG or $2 \mathrm{hPG}$ (=presented as $100 \%)$ ". 


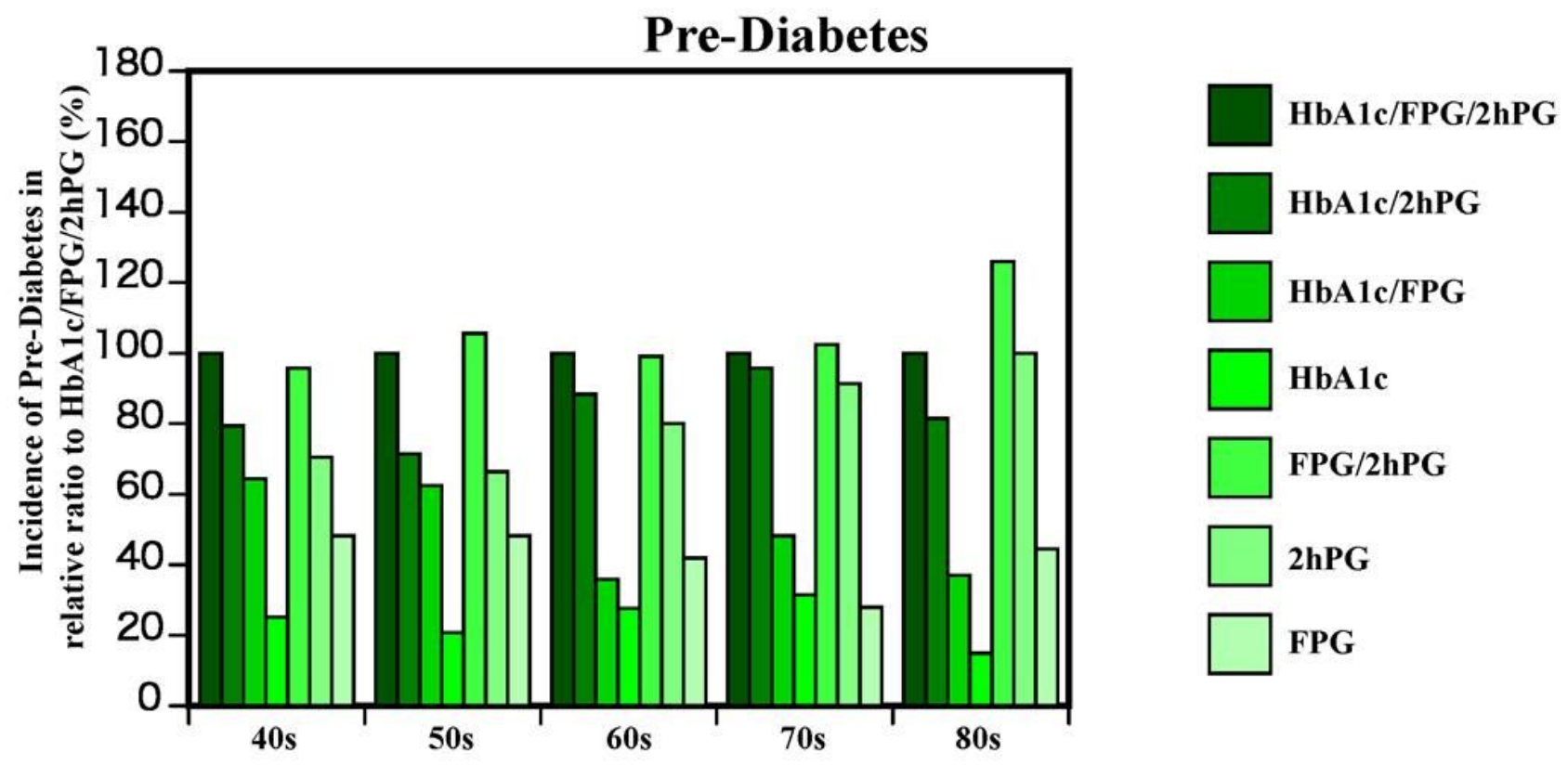

Figure 2

b. Incidence of Pre-Diabetes. $6.2<\mathrm{HbA} 1 \mathrm{c}<6.5 \%$ or $110<\mathrm{FPG}<126$ or $140<2 \mathrm{hPG}<200$ was determined as pre-diabetes. Each combination of these factors was compared to "the combination of either HbA1c or FPG or 2 hPG (=presented as $100 \%)$. 


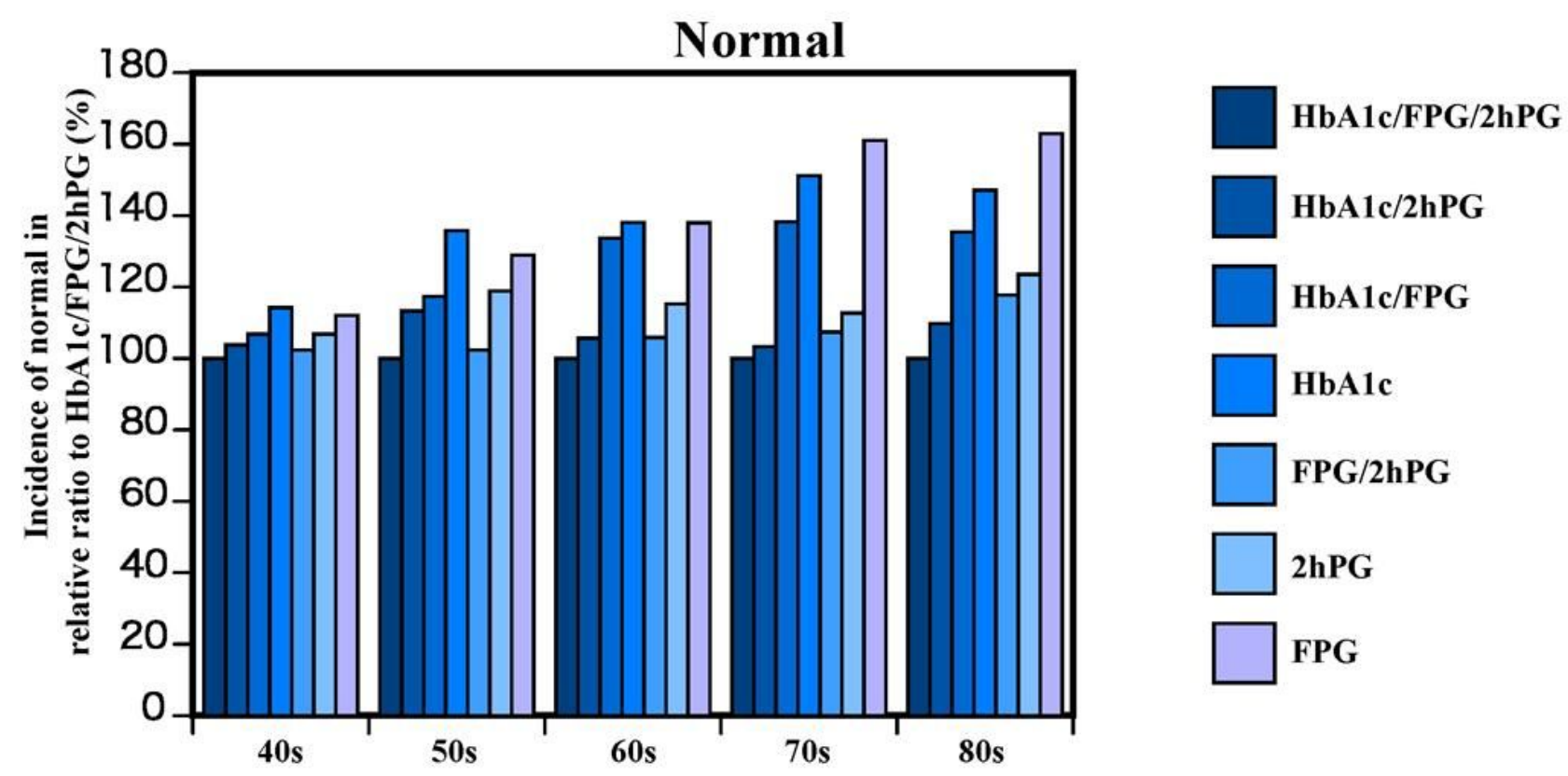

Figure 3

c. Incidence of Normal. The remaining subjects from the subtraction of "diabetes" and "pre-diabetes" was determined as normal. Each combination of these factors was compared to "the combination of either $\mathrm{HbA} 1 \mathrm{c}$ or FPG or $2 \mathrm{hPG}$ (=presented as $100 \%)$. 